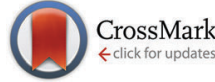

Cite this: Phys. Chem. Chem. Phys., 2015, 17, 1106

Received 26th August 2014, Accepted 15th October 2014

DOI: $10.1039 / c 4 c p 03835 e$

www.rsc.org/pccp

\section{Reverse hydrotropy by complex formation $\dagger$}

\author{
Kamil Wojciechowski, ${ }^{* a}$ Thomas Gutberlet, ${ }^{b}$ Vikram Singh Raghuwanshi ${ }^{\mathrm{b}}$ and \\ Ann Terry ${ }^{c}$
}

\begin{abstract}
Self-aggregation of three di- $N$-alkylated diaza-18-crown-6 ethers (ACEs) was studied in non-polar solvents. The three ACEs differed by the length of the alkyl chain: $n$-decyl (ACE-10), $n$-hexadecyl (ACE-16) and $n$-tetracosane (ACE-24). From the previously reported interfacial tension isotherms, the formation of reverse micelles was expected above ACE concentrations of $\sim 10^{-3} \mathrm{M}$. However, the water content analysis in conjunction with Dynamic Light Scattering (DLS), Fluorescence Correlation Spectroscopy (FCS) and ${ }^{1} \mathrm{H}$ NMR Diffusion Ordered Spectroscopy (DOSY) do not provide any clear proof of the existence of aggregates. Only the Small Angle Neutron Scattering (SANS) of concentrated toluene ACE solutions reveals the existence of small reverse micelles (probably ACE dimers forming small cages hosting 1-2 water molecules). On the other hand, spectrophotometric and fluorescence dye dissolution studies using eosin Y, tropaeolin $\bigcirc \bigcirc$ and methyl orange suggest that ACEs can dissolve these dyes without requiring the formation of aggregates. This discrepancy was interpreted assuming the dye-ACE complexation as the driving force for dye solubilisation, providing a possible mechanism of reverse hydrotropy ("lipotropy") in non-polar solvents. This example shows that special care should be taken when dye solubilisation is used to probe self-aggregation of an amphiphile in non-polar solvents. The amphiphile-dye complex formation might be responsible for false positive results and the aggregate formation should always be confirmed with other methods.
\end{abstract}

\section{Introduction}

The combination of a hydrophilic macrocyclic cavity (azacrown ether) with lipophilic $n$-alkyl chains makes alkylated azacrown ethers (ACEs) especially useful candidates for interfacial complexation and transport of metal ions in extraction-based techniques. The ion-recognition selectivity is determined by the size of the macrocyclic cavity and the topology of heteroatoms in the cavity (oxygen and nitrogen atoms in the present case, but others, e.g., sulphur, can also be employed). The long lipophilic alkyl chains, on the other hand, permit the solubility of an ACE molecule and its metal ion complex in non-polar solvents. The presence of both hydrophilic and lipophilic moieties in the same molecule imparts an amphiphilic character to the ACE molecules, which makes them efficient phase transfer catalysts e.g., for $\mathrm{Cu}(\mathrm{II})$ transport through Permeation Liquid Membranes (PLM). ${ }^{1}$ The di-decyl substituted ACE (ACE-10, see Fig. 1) has been extensively used as Cu(II) and $\mathrm{Pb}$ (II) ion carriers (in combination with lauric or palmitic acid) for environmental speciation studies. ${ }^{2}$

\footnotetext{
${ }^{a}$ Faculty of Chemistry, Warsaw University of Technology, Noakowskiego 3, o0-664 Warsaw, Poland. E-mail: kamil.wojciechowski@ch.pw.edu.pl

${ }^{b}$ Helmholtz-Zentrum Berlin für Materialien und Energie GmbH,

Hahn-Meitner-Platz 1, 14109 Berlin, Germany

${ }^{c}$ ISIS, STFC, Rutherford Appleton Laboratory, Harwell Oxford, Didcot, OX11 OQX, UK

$\dagger$ Dedicated to Professor Jacques Buffle on the occasion of his 70th birthday.
}

Amphiphilic molecules above their Critical Micelle Concentration (CMC) often self-aggregate to form normal or reverse micelles, depending on the polarity of the solvent and geometry of the molecule. The type of aggregates can be roughly predicted from the geometry of individual molecules using a packing parameter, $P$, as the criterion, ${ }^{3} P=\frac{V}{a_{0} l_{\mathrm{c}}}$, where $V$ is the alkyl chain volume, $a_{0}$ is the surface area, and $l_{\mathrm{c}}$ is the length of the alkyl chain. These simple geometric considerations impose a condition $P<1$ for the normal micelles (in polar solvents), and $P>1$ for the reverse micelles (in nonpolar solvents). For ACE molecules with alkyl chain lengths exceeding 10 carbon atoms (as employed in the current study), $P$ takes a value of about 0.5 , provided that the alkyl chains arrange perpendicularly to the azacrown plane. This would suggest a propensity of ACE to form normal micelles, if it only were soluble in water, which is not the case (for the shortest chain (i.e., the most water soluble), ACE-10, the solubility in water is as low as $(2.7 \pm 0.1) \times 10^{-5} \mathrm{M}$ at $25{ }^{\circ} \mathrm{C}$, ref. 4$)$.

On the other hand, the alkyl chains are likely capable of pivoting on tetrahedral nitrogen atoms, enabling the $P$ parameter to take higher values, even exceeding 1 . One could thus envisage the existence of reverse micelles of ACE in concentrated non-polar solutions.

Not all amphiphilic molecules possess, however, the ability to self-assemble, even though they can still significantly lower the surface tension. These (usually small) amphiphiles, 


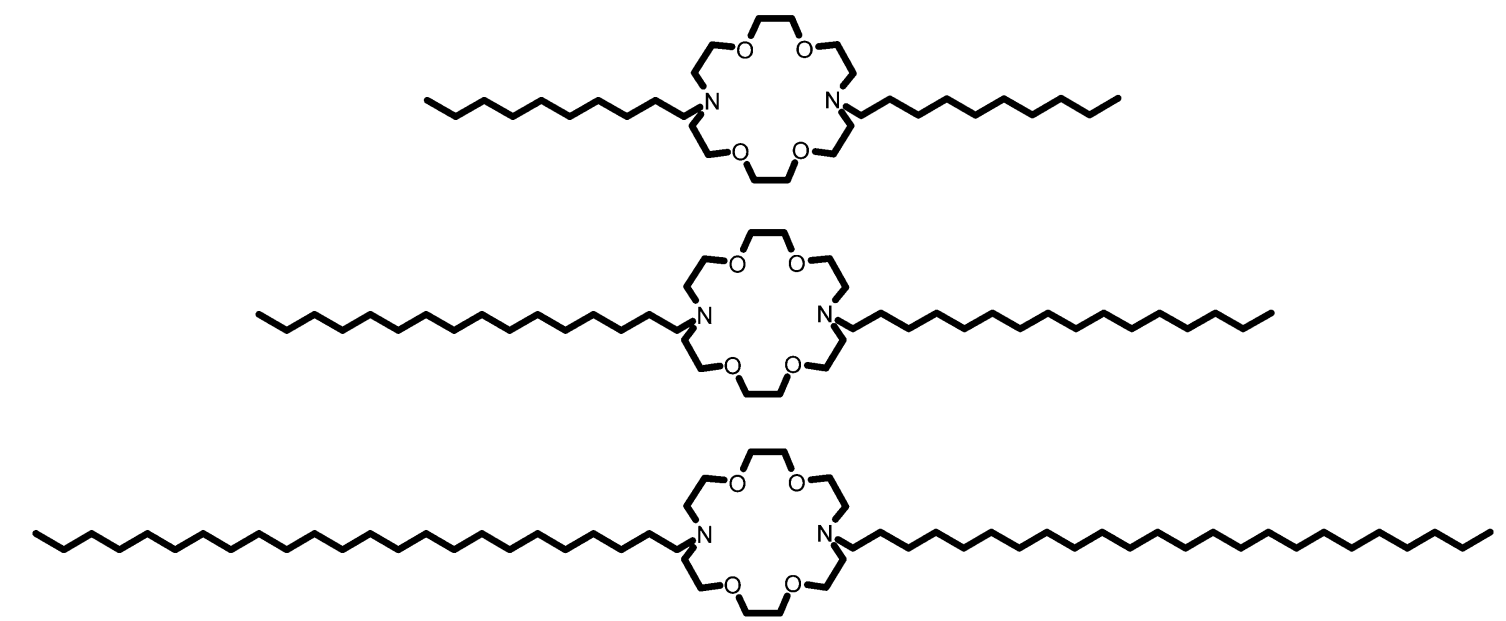

Fig. 1 Structures of alkylated azacrown ether amphiphiles used in the present study, ACE-10, ACE-16, and ACE-24.

called hydrotropes, find numerous industrial applications, for their ability to solubilise large amounts of hydrophobic substances in aqueous solutions. ${ }^{5,6}$ Similar to the CMC for surfactants, a Minimum Hydrotrope Concentration (MHC) can be defined, where macroscopic solution properties (e.g., surface tension) change abruptly, and the hydrotropic behaviour of a given solution commences. Even though it is almost one century since the first reports of hydrotropy by the German biochemist C. Neuberg (see ref. 5 for more details), the details of structures formed by hydrotropes in solutions are still missing. ${ }^{4,5}$ According to Eastoe et al., hydrotropy can be caused by either weak self-association, solvent structure making/ breaking by the hydrotropes, or complex formation between the hydrotrope and the solute. ${ }^{6}$

In analogy to the hydrotropes in aqueous solutions, poorly organised self-assembled structures might also probably develop in non-polar solvents. To the best of our knowledge, so far only one paper has been reported on their existence. Bauduin et al. observed "reverse hydrotropic (lipotropic)" behaviour of $n$-alcohols in alkanes ${ }^{7}$ and revealed that water and a hydrophilic dye (Orange II) can be solubilised in dodecane upon addition of short chain alcohols.

In this paper, we report on self-aggregation of the toluene-soluble ACE-10 ( $-\mathrm{C}_{10} \mathrm{H}_{21}$ alkyl chains) amphiphile and its two homologues with different alkyl chain lengths: $-\mathrm{C}_{16} \mathrm{H}_{33}$ and $-\mathrm{C}_{24} \mathrm{H}_{49}$ (ACE-16 and ACE-24, Fig. 1). We show that these amphiphiles are capable of effectively solubilising hydrophilic dyes. This solubilisation may take place either in the water pools of small reverse micelles, or in the organic phase by simple ACE-dye complex formation. These amphiphiles seem to provide an example of reverse hydrotropy ("lipotropy") only due to chemical interactions between an amphiphile and a hydrophilic molecule in non-polar solvents.

\section{Experimental}

\section{Chemicals}

$N, N^{\prime}$-Didecyldiaza-18-crown-6 ether (ACE-10) was obtained from Merck (Kryptofix ${ }^{\circledR}$ 22DD). $N, N^{\prime}$-Dihexadecyl-4,13-diaza-18-crown-6 (ACE-16) and $N, N^{\prime}$-ditetracosane-4,13-diaza-18-crown-6 (ACE-24) were prepared as described in ref. 8. 4-Morpholineethanesulfonic acid (MES) >99.5\%, methyl orange, tropaeolin OO and eosin Y were purchased from Sigma-Aldrich. The BODIPY FL C-12 fluorescent probe was obtained from Molecular Probes, USA. Freshly deionised water from a Millipore Simplicity UV system $\left(18.2 \times 10^{6} \Omega \mathrm{cm}^{-1}\right.$, irradiated with UV lamp) was used for the measurements. Toluene (puriss. p.a. ACS reagent for UV spectroscopy) was purchased from Sigma-Aldrich and purified by liquid-liquid extraction with water. For water solubilisation studies, toluene stored over molecular sieves (puriss, $\mathrm{H}_{2} \mathrm{O} \leq 0.005 \%$ ) from Sigma-Aldrich was used.

Deuterated solvents for ${ }^{1} \mathrm{H}$ NMR spectroscopy (toluene- $\mathrm{D}_{8}$, octane- $\mathrm{D}_{18}$, cyclohexane- $\mathrm{D}_{12}$, and methanol- $\mathrm{D}_{4}$ ) were purchased from Cambridge Isotope Laboratories, Inc (UK) and Armar AG (Switzerland). Deuterated toluene $(99.6 \%$ D) for SANS was purchased from Sigma-Aldrich and used without further purification. The chain-deuterated ACE-16 (d-ACE-16) was provided by BDG Synthesis (New Zealand).

\section{Measurements}

For the dye solubilisation study, the azacrown ethers were dissolved in toluene at concentrations between $3 \times 10^{-5} \mathrm{M}$ and $10^{-2} \mathrm{M}$ (for ACE-10 and ACE-16), and between $10^{-5} \mathrm{M}$ and $2 \times 10^{-3} \mathrm{M}$ (for ACE-24). $1 \mathrm{ml}$ of the toluene solution was handshaken with $1 \mathrm{ml}$ of the MES-buffered aqueous solution $(0.01 \mathrm{M}$, $\mathrm{pH}$ 6.0) of one of the dyes: eosin $\mathrm{Y}\left(10^{-5} \mathrm{M}\right)$, tropaeolin OO $\left(3 \times 10^{-5} \mathrm{M}\right)$, and methyl orange $\left(3 \times 10^{-5} \mathrm{M}\right)$. After the two phases had separated, the toluene phase was analysed using a Jasco FP 750 spectrofluorometer (for eosin Y, $\lambda_{\text {exc }}=310 \mathrm{~nm}, \lambda_{\mathrm{em}}=558 \mathrm{~nm}$ ) or a Zeiss Specord S600 spectrophotometer (for methyl orange, $\lambda_{\text {max }}=414 \mathrm{~nm}$ and for tropaeolin OO, $\lambda_{\text {max }}=408 \mathrm{~nm}$ ).

The water solubilisation was investigated by adding water from a micropipette to the initially dry toluene solution of ACE-10 $\left(10^{-4} \mathrm{M}, 10^{-3} \mathrm{M}\right.$, and $\left.10^{-2} \mathrm{M}\right)$ over solid eosin $\mathrm{Y}$. The solid eosin $\mathrm{Y}$ was deposited from a methanol solution $\left(1 \mathrm{mg} \mathrm{ml}^{-1}\right)$, with the solvent being left to evaporate prior to toluene addition. After gentle shaking for $1 \mathrm{~min}$ of the closed vial the solution was taken for spectrofluorometric analysis as described above. 
The water content in toluene solutions of ACE-10 after equilibration with water and aqueous solutions of eosin $\mathrm{Y}$ was measured using a Mettler Toledo Karl Fischer C30 titrator. The analysis was performed for samples left to equilibrate for at least 7 days.

Dynamic Light Scattering (DLS) measurements were performed using a Zetasizer HS 3000 at $25{ }^{\circ} \mathrm{C} .5 \mathrm{ml}$ of $10^{-4} \mathrm{M}$, $10^{-3} \mathrm{M}$ and $10^{-2} \mathrm{M}$ solutions of ACE-10 in toluene were handshaken with $5 \mathrm{ml}$ of water and $3 \times 10^{-5} \mathrm{M}$ eosin $\mathrm{Y}$ solution in MES (0.01 M, pH 6.0) and left to phase-separate for at least $2 \mathrm{~h}$. Some samples were also obtained by gently pouring the toluene phase on top of the aqueous one, with as little mechanical disturbance as possible. Both aqueous and toluene solutions were filtered prior to mixing them.

Fluorescence Correlation Spectroscopy measurements of ACE-10 were performed in $2.3 \times 10^{-3} \mathrm{M}$ solutions in toluene/ hexane (1/1) mixtures using a ConfoCor Axiovert 135 TV microscope (Carl Zeiss). An Ar ion laser was used (488 nm) and a BODIPY FL C-12 fluorescent probe was employed to highlight the possible aggregates.

A Fluorescence Confocal Microscope (Olympus Fluoview FV10i) equipped with $10 \times$ and $60 \times$ objectives was used to search for potential water-filled aggregates in toluene solutions of ACE-10 $\left(10^{-2} \mathrm{M}\right)$ after equilibrating it against the aqueous solution of eosin $\mathrm{Y}\left(3 \times 10^{-5} \mathrm{M}\right)$ in MES buffer $(\mathrm{pH} 6)$ for 1 day. A drop of the pink-coloured toluene phase was placed on a microscopic slide and covered with another glass slide prior to analysis.

${ }^{1} \mathrm{H}$ NMR Diffusion Ordered Spectroscopy (DOSY) was performed using a Bruker $500 \mathrm{MHz}$ at $30{ }^{\circ} \mathrm{C}$ (the temperature was increased to facilitate temperature stabilisation during the NMR measurements) in solutions of ACE-10 at two concentrations of $1 \times 10^{-2} \mathrm{M}$ and $6 \times 10^{-2} \mathrm{M}$ in the following deuterated solvents: toluene- $\mathrm{D}_{8}$, octane- $\mathrm{D}_{18}$, cyclohexane- $\mathrm{D}_{12}$, and methanol- $\mathrm{D}_{4}$.

Small Angle Neutron Scattering (SANS) experiments were performed on the LOQ small-angle neutron scattering instrument $^{9}$ at the ISIS Pulsed Neutron Source (STFC Rutherford Appleton Laboratory, Didcot, UK). LOQ is a fixed-geometry "white beam" time-of-flight instrument which utilizes neutrons with wavelengths, $\lambda$, between 0.2 and $1 \mathrm{~nm}$. SANS spectra were recorded in the range of $0.01<q<0.25 \AA^{-1}$ using a sample-todetector distance of $4.15 \mathrm{~m}$. Each raw scattering data set was then corrected for the detector efficiencies, sample transmission and background scattering, and placed on an absolute scale $\left(\mathrm{cm}^{-1}\right)$ using the scattering from a standard sample. Mantid software on LOQ (http://www.small-angle.ac.uk/smallangle/Software/MantidPlot.html) was used for this purpose. Protonated ACE-16 (h-ACE-16) and deuterated ACE-16 (d-ACE-16) were dissolved in deuterated and protonated toluene solutions, respectively. For the protonated azacrown ether two concentrations were used: $10^{-2} \mathrm{M}$ and $4 \times 10^{-2} \mathrm{M}$. For the deuterated derivative the solubility in both protonated and deuterated toluene solutions prevented any measurements above $10^{-2} \mathrm{M}$. The solutions were measured in quartz cells, either as such, or after shaking with $\mathrm{D}_{2} \mathrm{O}$ or $\mathrm{H}_{2} \mathrm{O}$ (when the two phases had separated).

\section{Results}

The interfacial tension isotherms described in our previous report display inflection points typical for the CMC of around $10^{-3} \mathrm{M}$ for all three alkylated azacrown ethers (ACE-10, ACE-16, and ACE-24). ${ }^{10}$ Since the azacrown ethers remain predominantly in the toluene phase and the packing parameter, $P$, can take values above 1 , reverse micelles could be expected to form, with hydrophilic parts (azacrown ether moiety) buried in the cores of the aggregates, possibly filled with water. ${ }^{11}$ It should be borne in mind, however, that the drop shape analysis method employed in the previous study is not well suited for determination of values of interfacial tension below $1 \mathrm{mN} \mathrm{m}^{-1}$. For the latter case, the effect of gravity is too high and the drops become highly elongated, which may lead to substantial errors during interfacial tension determination. Thus, to shed more light on possible formation and structure of the ACE aggregates, UV/Vis spectrophotometry and spectrofluorimetry were first employed, complemented by scattering techniques, magnetic resonance and coulometric determination of the water content in the toluene phase, as described below.

\section{Dye solubilisation in an initially dry ACE-10 toluene solution}

The first experiment comprised the slow addition of water to the initially dry toluene solution of ACE-10 in contact with solid eosin Y. Since the latter is not soluble in dry toluene, in the absence of any water-mediated ACE-dye interactions, the fluorescence in the organic phase could only be triggered by its solubilisation in the aqueous phase dispersed in toluene. Note that the addition of even high amounts of ACE- $10\left(10^{-2} \mathrm{M}\right)$ to the dry toluene phase does not produce any significant fluorescence of the toluene phase in the absence of water. On the other hand, the UV/Vis spectra of the aqueous solution of the dyes $\left(3 \times 10^{-5} \mathrm{M}\right)$ do not change upon addition of saturated ACE-10 aqueous solution $\left(2.7 \times 10^{-5} \mathrm{M}\right)$, confirming that no dye-ACE complexation takes place in the aqueous phase.

The intensity of the eosin $\mathrm{Y}$ emission peak in the toluene phase as a function of water concentration, $\left[\mathrm{H}_{2} \mathrm{O}\right]$ (upper $x$-axis), for three different ACE-10 concentrations is shown in Fig. 2. For comparison, the lower $x$-axis is scaled with the parameter $W$, expressing the ratio of water to surfactant concentrations $\left(W=\left[\mathrm{H}_{2} \mathrm{O}\right] /[\mathrm{ACE}-10]\right)$, as it is commonly presented for reverse micelles. Clearly, with the increase of both water and ACE-10 concentrations in the toluene phase, the emission intensity also increases. The three curves shown in Fig. 2 display inflection points at the same bulk concentration of water (although not the same $W$ ), above which the fluorescence intensity stabilises at the level depending on the total concentration of ACE-10. This suggests that the water load (but not that of the dye) in toluene might stabilise at the level of $\sim 0.06 \%\left(\sim 3 \times 10^{-2} \mathrm{M}\right)$, independent of the ACE-10 concentration.

The $W$ parameter, for which a good correlation was found with the size of the aggregates, ${ }^{12}$ so far has been almost exclusively applied to the inverted micelles formed by sodium bis(2-ethylhexyl)sulfosuccinate (AOT). However, this approach clearly does not work for the alkylated azacrown ether employed in the present 


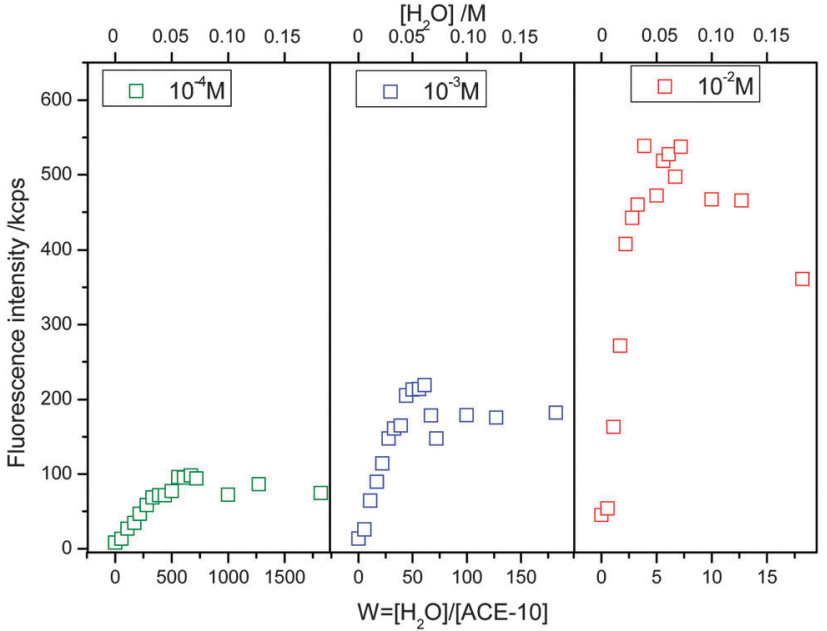

Fig. 2 Eosin $Y$ fluorescence intensity of the toluene phase containing ACE-10 at three concentrations $\left(10^{-4} \mathrm{M}, 10^{-3} \mathrm{M}\right.$ and $\left.10^{-2} \mathrm{M}\right)$ as a function of water concentration. Upper $x$-axis scale: water concentration in toluene; lower $x$-axis: $W$ parameter (see the text).

study, as evident from Fig. 2 (bottom $x$-axis). Upon increase of the ACE-10 concentration, the value of the $W$ parameter corresponding to the maximum fluorescence intensity decreases, which would imply that the size of the aggregates decreases with an increase in ACE concentration. This is, however, counterintuitive and contradicts another observation from the same experiment that the dye fluorescence intensity in toluene increases with an increase of ACE concentration. The inconsistency of the picture given by analysis of the $W$ parameter suggests that the alkylated azacrown ethers may not necessarily form typical reverse micelles with the size correlated with the amount of water available.

In order to confirm the observations from the fluorescence probe solubilisation concerning the weak dependency of water solubility in toluene on ACE-10 concentration, the water content in equilibrated toluene solutions of ACE- $10\left(10^{-4} \mathrm{M}, 10^{-3} \mathrm{M}\right.$, $10^{-2} \mathrm{M}$, and $5 \times 10^{-2} \mathrm{M}$ ) was determined. For this purpose, the toluene phase after contact with both water and aqueous solutions of eosin $\mathrm{Y}\left(3 \times 10^{-5} \mathrm{M}\right.$ solution in MES buffer $0.01 \mathrm{M}$, $\mathrm{pH}$ 6.0) was analysed using the Karl-Fischer method. These values (Table 1) are in good agreement with the water concentrations corresponding to the saturation of the fluorescence signal in the water solubilisation experiments. In all cases, no significant differences were observed between samples equilibrated with water and eosin Y, neither between the samples that were vigorously shaken and those left to equilibrate under quiescent conditions (for the same ACE-10 concentration). A slightly increased water content in toluene can be detected only above an ACE-10 concentration of $10^{-2} \mathrm{M}$.

Table 1 Water content in water-saturated toluene solutions of ACE-10, determined using the Karl-Fischer titration

$c_{\mathrm{ACE}-10} / \mathrm{M} \quad 1.0 \times 10^{-5} \quad 1.0 \times 10^{-4} \quad 1.0 \times 10^{-3} \quad 1.0 \times 10^{-2} \quad 5.0 \times 10^{-2}$ $c_{\mathrm{H}_{2} \mathrm{O}} / \mathrm{M} \quad 3.1 \times 10^{-2} 3.1 \times 10^{-2} 3.2 \times 10^{-2} 3.7 \times 10^{-2} \quad 4.7 \times 10^{-2}$
The fact that the water content increases only slightly with an increase of ACE concentration in toluene solutions is consistent with the presence of only small water-filled aggregates or even their absence. The water content started to increase appreciably only when the ACE concentrations approached that of the initial solubility of water in toluene. At the highest ACE concentration the number of water molecules is equal to that of ACE in the toluene phase. It should be stressed that this concentration by far exceeds the apparent CMC obtained from the interfacial tension measurements. Consequently, the existence of AOT-like water-filled aggregates in ACE solutions in toluene is highly questionable.

\section{Dye solubilisation in water-saturated ACE-10, ACE-16 and ACE-24 toluene solutions}

In order to compare the dye solubilisation behaviour of the three homologous ACEs, their toluene solutions were equilibrated with the pH-buffered ( $\mathrm{pH}$ 6) aqueous solutions of the hydrophilic optical dyes: one fluorescent (eosin Y), and two spectrophotometric (tropaeolin OO and methyl orange). After phase separation, the UV/Vis absorption and fluorescence spectra of the coloured toluene phase were recorded. Interestingly, the samples that were not shaken, but prepared by gently pouring the toluene phase on top of the aqueous dye solution, also showed coloration of the toluene phase. This coloration persists even after passing through a $0.45 \mu \mathrm{m}$ PTFE filter or centrifugation at $18000 \mathrm{rpm}$ for $30 \mathrm{~min}$. It should be noted that pure toluene equilibrated with the probes' solutions displays negligible absorption/emission.

The dependence of the absorbance/emission at the respective peak maxima for each ACE as a function of its concentration in toluene is shown in Fig. 3 (for the sake of comparison, also the interfacial tension isotherms, taken fromref. 10 are shown). The data start to show evidence of the presence of the spectrophotometric dyes (tropaeolin OO and methyl orange) in the toluene phase above an ACE concentration of $10^{-4} \mathrm{M}$, while the more sensitive fluorescence dye (eosin Y) is evident even at concentrations one order of magnitude lower. A plateau and even a maximum (for ACE-16) in the dye absorption/emission are observed around a concentration of $10^{-3} \mathrm{M}$, which would coincide with the CMC from the interfacial tension data. For the reasons addressed below, the latter concentration will be hereafter referred to as "apparent CMC". The intensity of the light absorption/emission increases from ACE-10 to ACE-16, and finally decreases for the longest chain derivative ACE-24.

Although the critical micelle concentration for reverse micelle formation is not as abrupt as for normal micelles, in some cases a range of concentrations where the aggregates start to appear can be defined ("operational CMC"). ${ }^{17-19}$ The comparison of the apparent CMC values obtained from the above interfacial tension studies with those from the optical probes shows that the dye solubilisation in toluene solutions containing ACE is definitely not a well-defined process. Instead, a continuous dye dissolution takes place in the toluene phase starting around bulk concentrations as low as $10^{-5} \mathrm{M}$, corresponding to near zero surface coverage (Fig. 3 and ref. 10). With an increase in the 


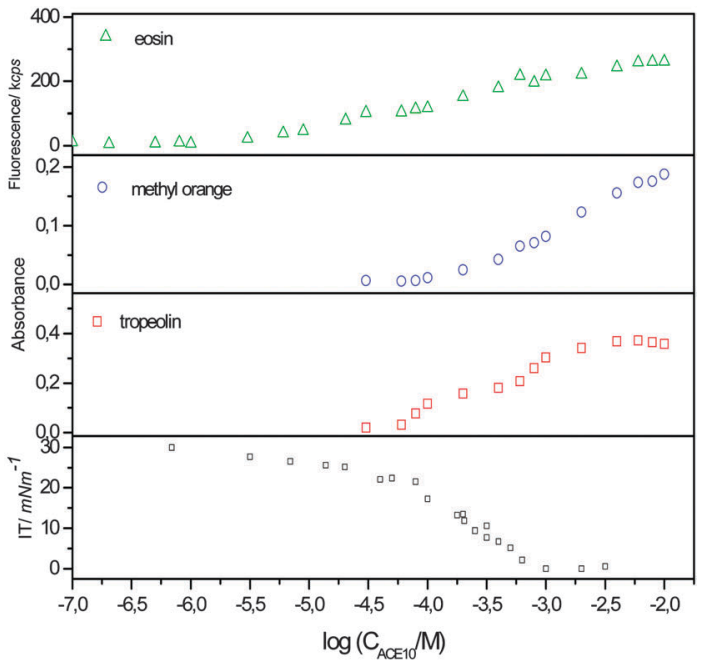

a)

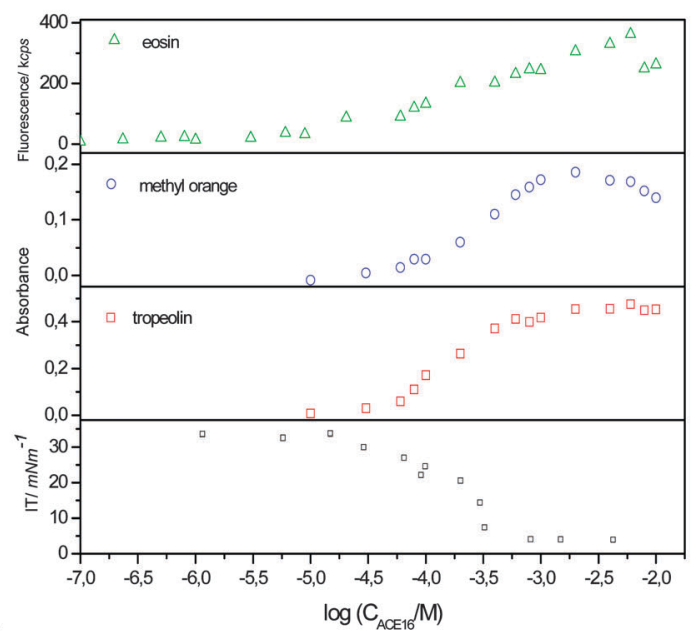

b)

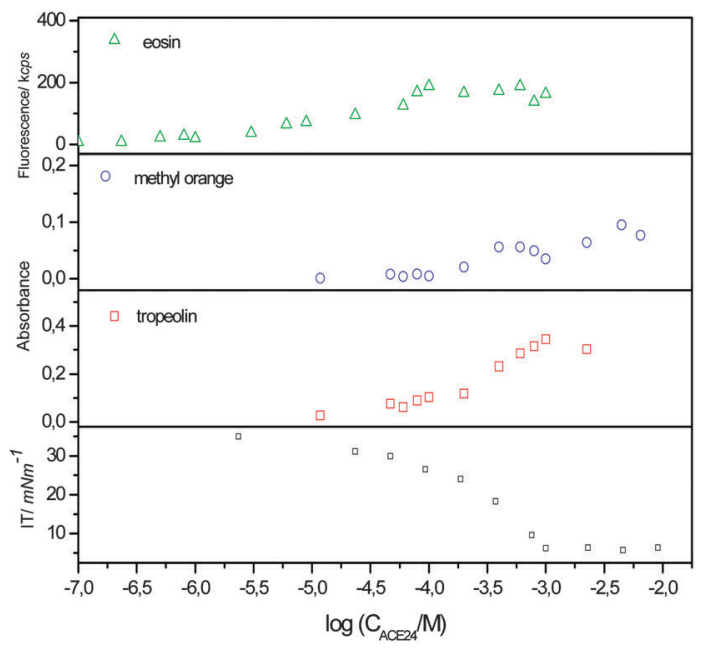

c)

Fig. 3 ACE concentration dependence of absorbance (methyl orange, $\lambda_{\max }=414 \mathrm{~nm}$ and tropaeolin $00, \lambda_{\text {exc }}=408 \mathrm{~nm}$ ) and emission (eosin $Y, \lambda_{\mathrm{exc}}=310 \mathrm{~nm}, \lambda_{\mathrm{em}}=558 \mathrm{~nm}$ ) of the toluene phase for (a) ACE-10, (b) ACE-16 and (c) ACE-24. The interfacial tension isotherms are reproduced from ref. 10.
ACE concentration the fluorescence intensity reaches a maximum around $10^{-3} \mathrm{M}$, where also a maximum surface coverage is reached, based on the interfacial tension results. The emission intensity and absorbance of light by the probes for the three homologous ACEs reach a maximum for the middle-chain ACE-16 derivative, suggesting that the ability to solubilize hydrophilic species may depend (albeit weakly) on a balance between the hydrophilic and lipophilic groups of the azacrown ether amphiphiles.

Exploring ACE-10 aggregation in the toluene phase (FCS, CFM, DLS, and ${ }^{1} \mathrm{H}$ NMR DOSY)

In order to acquire information regarding the existence of ACE-10 aggregates in the organic phase, ${ }^{1} \mathrm{H}$ NMR DOSY spectra were recorded in four different deuterated solvents (toluene- $\mathrm{D}_{8}$, octane- $\mathrm{D}_{18}$, cyclohexane- $\mathrm{D}_{12}$, and methanol- $\left.\mathrm{D}_{4}\right)$. The DOSY technique provides information on self-diffusion coefficients of the protons associated with each individual signal in the NMR spectrum, and has been used successfully to study the self-aggregation process in various solvents. ${ }^{13-15}$ The measurements were performed at ACE concentrations sixty times above the apparent $\mathrm{CMC}$ (at $6 \times 10^{-2} \mathrm{M}$ ), in order to ensure that the majority of the ACE molecules would exist in the aggregated state. In all cases a single group of signals was observed in the DOSY spectrum for all protons of the ACE-10 molecule, with the values of self-diffusion coefficient, $D$, varying between $5 \times 10^{-10} \mathrm{~m}^{2} \mathrm{~s}^{-1}$ and $1 \times 10^{-9} \mathrm{~m}^{2} \mathrm{~s}^{-1}$, depending on the solvent. The corresponding hydrodynamic radii, obtained from the StokesEinstein equation using the appropriate solvent viscosities, gave values in the range 4.0-4.3 $\AA$, i.e. the expected size of a monomeric ACE-10 molecule. The ${ }^{1} \mathrm{H}$ DOSY spectra of ACE-10 in toluene and octane were additionally recorded in water- and eosin Y solutionsaturated solvents, but the results were the same, except for the presence of an additional peak at $\delta=5.0-5.6 \mathrm{ppm}$, assigned to $\mathrm{H}_{2} \mathrm{O}$ shifted downfield with respect to the expected value for bulk water. The analysis of this peak provides a rather broad distribution of the diffusion coefficient of water in the ACE-10 toluene solution, $D=(2.0-2.5) \times 10^{-9} \mathrm{~m}^{2} \mathrm{~s}^{-1}$, which is close to the values of bulk $\mathrm{H}_{2} \mathrm{O} .{ }^{16}$ From the integrated area of the water peak, the molar ratio of water/ACE-10 can be estimated to be $\sim 1.5$, in good agreement with the results of the Karl-Fischer titration results (Table 1). Thus, the peak can be assigned to the water molecules solubilised in the nonpolar phase, hydrating the ACE headgroups.

In agreement with DOSY, also Confocal Fluorescence Microscopy (CFM) did not reveal any aggregates containing eosin Y in the toluene phase, suggesting that the coloration of the toluene ACE solutions upon contact with the dye is not associated with any aggregated structures of the size detectable with the setup employed (few $\mu \mathrm{m}$ ). To complement the CFM and DOSY results, further attempts were made with Fluorescence Correlation Spectroscopy (FCS) and Dynamic Light Scattering (DLS) to probe the diffusion behaviour of the possible aggregates. For the former, a lipophilic fluorescent probe, BODIPY FL-C12, was employed. The probe applied contains a fatty acid moiety, which is expected to bind to the ACE-10 molecules, ${ }^{17}$ and thus enables 
detection of aggregates. The diffusion coefficient of the probe in $2.3 \times 10^{-3} \mathrm{M}$ ACE-10 solution in toluene/hexane (50/50) was, however, very close to that obtained for the bare probe in the same solvent $\left(2.4 \times 10^{-10} \mathrm{~m}^{2} \mathrm{~s}^{-1}\right.$ vs. $\left.2.7 \times 10^{-10} \mathrm{~m}^{2} \mathrm{~s}^{-1}\right)$. This shows that the probe does not get incorporated into any larger structure when ACE-10 is present in the solution.

The ACE-10 solutions in toluene equilibrated with water or aqueous eosin Y solutions (with and without shaking) displayed strong light scattering and the analysis of the correlation function in the DLS experiments pointed to the presence of large objects of the range of $1 \mu \mathrm{m}$ in diameter. However, the scattered light intensity dropped to almost zero upon filtration of the water-saturated ACE-10 solutions through a $0.22 \mu \mathrm{m}$ filter or when the toluene phase was left to equilibrate for several days. Similarly, when dry toluene was used to prepare the ACE-10 solutions, the light scattering was very weak. This points to the formation of a water-in-oil emulsion with $>$ few hundred nm diameter water droplets as a possible origin of the intense light scattering from freshly prepared ACE-10 solutions. The kinetic stability of the emulsions was dependent on the ACE-10 concentration: for $c_{\mathrm{ACE}-10}$ below $10^{-3} \mathrm{M}$ the intensity of the scattered light was decaying on the time scale of the DLS measurement (minutes), while for $c_{\mathrm{ACE}-10}=1 \times 10^{-2} \mathrm{M}$ the readings were stable for at least two days.

The three spectroscopic and scattering techniques, FCS, DOSY and DLS, provided arguments against the presence of ACE reverse micelles in the toluene phase. DLS and FCS detected only large droplets in water-saturated solutions at higher ACE concentrations. Their size is, however, far too large for typical reverse micelles, and they are more likely aqueous drops of water-in-oil emulsions, formed due to the low interfacial tension (see Fig. 3). The persistence of the pink coloration after filtration of the toluene ACE solutions pre-equilibrated with eosin Y (despite a complete disappearance of the DLS signal) would indicate that if the dye is associated with the ACE aggregates, the latter would have to be quite small, below the detection limit of the DLS instrument. Under optimum conditions DLS is capable of detecting objects as low as $2 \mathrm{~nm}$, but with the low difference in refractive indices (low contrast) between the organic solvent and ACE, combined with low amount of water (possibly present only as hydration water, see above) the small ACE aggregates could simply be undetectable for DLS.

\section{ACE-16 aggregation in the toluene phase probed with SANS}

Small Angle Neutron Scattering (SANS) was employed to confirm or exclude the existence of ACE aggregates. For this purpose, ACE-16 was dissolved in either protonated or deuterated toluene, with small amount of $\mathrm{H}_{2} \mathrm{O}$ or $\mathrm{D}_{2} \mathrm{O}$ added. Both, fully protonated and alkyl chain-deuterated ACE-16 analogues were employed. The combination of protonated and deuterated substances was chosen to maximise the neutron scattering contrast necessary to detect ACE-16 aggregates. The azacrown ether concentration in each sample was at least ten times higher than the apparent CMC of ACE-16 $\left(1 \times 10^{-2} \mathrm{M}\right.$ and $\left.4 \times 10^{-2} \mathrm{M}\right)$.

The SANS spectrum obtained for the combination of the alkyl chain deuterated ACE-16 and protonated toluene (Fig. 4)

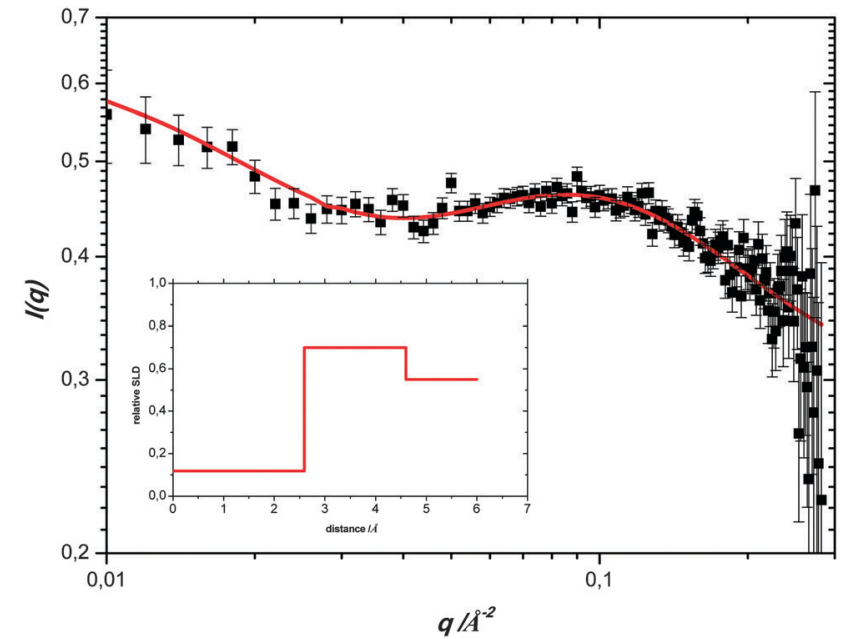

Fig. 4 SANS profile of deuterated ACE-16 in protonated toluene. The red line shows a fit of a spherical core shell structure with $9.2 \AA$ diameter. Inset: the corresponding spherical core shell model with relative neutron SLD (scattering length density profile), normalised to the SLD of the solvent.

shows no signature of compact micellar structures in the $q$-range probed. A simple spherical core shell model (ref. https://kur.web. psi.ch/sans1/SANSSoft/sasfit.html) applied to fit the data resulted in a soft micellar structure with a diameter of about $9.2 \AA$ (Fig. 4). The inner core of this structure exhibits a diameter of about $5 \AA$ and a shell thickness of $2 \AA$. These inverse micelles possibly aggregate in a loose percolation chain giving rise to larger structures causing the increase in scattering at low $q .{ }^{18}$

The best-fit parameters of the SANS data (Table 2) are consistent with core-shell type aggregates with the core representing the azacrown ether headgroups possibly hydrated with water. The hydrophilic, headgroup-dominated core of the reverse micelles, as determined by SANS, is shielded from the non-polar exterior by the shell of the alkyl chains connected to the headgroups. The shell thickness of $2 \AA$ would suggest significant intermixing between the chains and the solvating toluene molecules, being much smaller than the theoretical thickness of straight $n-\mathrm{C}_{16} \mathrm{H}_{33}$ alkyl chains (21.7 ̊). Given their size, these aggregates are likely to be the ACE-16 dimers forming hydrophilic cages capable of hosting 1-2 water molecules inside. The increase in scattering intensity at low $q$ may suggest that these dimers could further aggregate into a loose percolation chain and form a weakly organised longer-range structure, in analogy to that proposed by Neuman and Ibrahim ${ }^{19}$ and Bauduin et al. ${ }^{7}$ for other amphiphiles. A "chain-like" assembly of stacking ACE molecules can be easily imagined based on their solid state behaviour. ${ }^{20-23}$

Table 2 SANS fitting parameters

\begin{tabular}{lll}
\hline & d-ACE/h-toluene & h-ACE/d-toluene \\
\hline Total radius $/ \AA$ & $4.6 \pm 0.8$ & $4.6 \pm 0.8$ \\
Core radius $/ \AA$ & $2.6 \pm 0.5$ & $2.6 \pm 0.5$ \\
SLD core $/ 10^{-6} \AA^{-2}$ & 0.12 & 0.18 \\
SLD shell $/ 10^{-6} \AA^{-2}$ & 0.70 & 3.50 \\
SLD solvent $/ 10^{-6} \AA^{-2}$ & 0.55 & 5.40 \\
Polydispersity & 0.62 & 0.63
\end{tabular}


A 1D-water channel reported by Fromm et al. ${ }^{22}$ in the solid state could even explain the low water-to-ACE molar ratio $(\leq 1$, see Table 1).

Nevertheless, the amount of water present in toluene solutions of ACE is probably only sufficient to solvate the ACE headgroups and thus is not available for the hydrophilic dyes solubilised in ACE solutions. As argued above, the picture of small, practically waterfree reverse micelles obtained from the SANS data cannot explain the capacity of ACE to dissolve hydrophilic water-soluble dyes, i.e., their reverse hydrotropic ("lipotropic") behaviour.

\section{Discussion}

The results described above prove that the ACE molecules do not significantly aggregate in non-polar solvents. However, the question of what drives the dye solubilisation remains still unanswered. Below we argue that the observed lipotropic activity might be in fact related to short-range chemical interactions between ACE and the dyes. The ACE headgroup contains electronrich oxygen and nitrogen atoms, hence it is potentially capable of coordinating cations or accepting hydrogen bonds. The watersoluble ACE analogues (with both nitrogen atoms methylated, ACE-1) were reported to form complexes with mono- and divalent metal ions. In water, the two nitrogen atoms of ACE- 1 can be protonated, with the logarithm of the respective protonation constants of 9.5 and 7.5. ${ }^{24}$ However, because of a low dielectric constant, the long-chain ACE molecules are probably not protonated in toluene (even saturated with water). For the same reason, the complexes of ACE with copper(II) ions (but not with sodium) can exist in toluene only when a lipophilic anion is present (provided e.g., by a fatty acid). ${ }^{1}$ Consequently, formation of complexes with the anionic dyes would require concomitant binding of protons or sodium ions. This is not expected from the available data on the complex formation ability of azacrown ethers. ${ }^{24}$ Despite this, the dyedissolving capacity correlates better with the ACE concentration than with the amount of water solubilised in toluene (the fluorescence intensity due to solubilisation of eosin $\mathrm{Y}$ increases in the range of ACE- 10 concentration of $10^{-4} \mathrm{M}-10^{-2} \mathrm{M}$ by a factor of five, while the water content increases by only $50 \%$ in this range of concentration). This may suggest that an ACE-dye complex could be formed in the biphasic systems employed in this study. Consequently, the curves in Fig. 3 could be quantitatively interpreted as simple ACE-dye binding curves. In fact, they can be well fitted with a partitioning-complexation (i.e. extraction) model, according to the following equation:

$$
\begin{gathered}
\mathrm{ACE}_{\mathrm{tol}}+\mathrm{DYE}_{\mathrm{aq}} \leftrightarrow \mathrm{ACE}-\mathrm{DYE} \mathrm{tol}_{\mathrm{tol}} \\
K_{\mathrm{ex}}=[\mathrm{ACE}-\mathrm{DYE}]_{\mathrm{tol}} /[\mathrm{ACE}]_{\mathrm{tol}} \times[\mathrm{DYE}]_{\mathrm{aq}}
\end{gathered}
$$

where "ACE", "DYE" and "ACE-DYE" stand for the alkylated azacrown ether (ACE-10, ACE-16, and ACE-24), optical probe (eosin Y, tropaeolin OO, and methyl orange), and their complex, respectively. The subscripts "tol" and "aq" refer to the toluene and aqueous phases, respectively.

The corresponding extraction constants obtained by a nonlinear fitting procedure using DynaFit 3 software ${ }^{25}$ are collected
Table 3 Best-fit values of logarithms of equilibrium constants, $\log \left(K_{\mathrm{ex}} / \mathrm{M}^{-1}\right)$, for the extraction of optical probes to the toluene phase by ACEs (eqn (1))

\begin{tabular}{llll}
\hline & \multicolumn{1}{l}{$\log \left(K_{\mathrm{ex}} / \mathrm{M}^{-1}\right)$} & & \\
\cline { 2 - 4 } Optical probe & ACE-10 & ACE-16 & ACE-24 \\
\hline Methyl orange & $2.81 \pm 0.03$ & $3.29 \pm 0.08$ & $3.26 \pm 0.16$ \\
Tropaeolin OO & $3.46 \pm 0.06$ & $3.77 \pm 0.06$ & $3.35 \pm 0.06$ \\
Eosin Y & $4.11 \pm 0.07$ & $3.82 \pm 0.08$ & $5.14 \pm 0.18$ \\
\hline
\end{tabular}

in Table 3. The $\log K_{\mathrm{ex}}$ values lie in a range of typical weak-tomedium strength molecular complexes held by non-covalent interactions. The main contribution to these interactions probably comes from the azacrown part, the alkyl chains providing only weak additional van der Waals attraction with the alkyl chains of the dyes. It is thus not surprising that the $\log K_{\text {ex }}$ values do not depend significantly on the length of alkyl chains in the studied ACE homologues.

The good quality of the fits prompts us to speculate that the dye solubilisation by ACEs used in this study is not related to any self-aggregation of ACEs, even though the latter takes place to some limited extent. Instead, a simple 1:1 complexation between ACEs and the dyes can explain the experimental observations of the reverse hydrotropic ("lipotropic") behaviour of the azacrown ethers, as well as the poor water solubilisation efficiency and strange behaviour of the $W$ parameter. The latter is simply not relevant in the present system, since no microemulsion is formed (as proved by SANS). This example shows that the dye solubilisation tests can sometimes provide misleading information concerning (reverse) micelles formation and structure. Thus, in the absence of other evidence, e.g., from scattering experiments, the dye solubilisation results in non-polar solvents should be treated with care. On the other hand, the present example provides one possible explanation of the mechanism of "lipotropic" behaviour, where hydrophilic molecules (e.g., dyes) can be solubilised in non-polar solvents by simple complexation with an amphiphile. As shown using this example, the complex formation may be independent of the state of aggregation of the amphiphile, hence the differences in the CMC obtained from dye solubilisation and the interfacial tension.

\section{Conclusions}

A combination of spectroscopic and scattering techniques enabled us to show that the alkylated azacrown ethers, dodecyl-, dihexadecane- and ditetracosane-diaza-18-crown-6 ethers (ACE-10, ACE-16 and ACE-24), can form only small ( $\sim 1 \mathrm{~nm}$ in diameter) reverse micelles, containing small amount of water (water-to-ACE ratio does not exceed one). These amphiphiles are also capable of solubilising hydrophilic dye molecules (eosin Y, tropaeolin OO and methyl orange), thus behaving effectively as reverse hydrotropes ("lipotropes"). However, the dye molecules do not seem to be solubilised in the aqueous core of the ACE reverse micelles. Instead, they probably form 1:1 complexes by coordination to the polar heads of the ACE molecules. As a result, the dye solubilisation can only provide apparent CMC values, not 
coinciding with those from the interfacial tension isotherms. The example described in this contribution shows that dye solubilisation as a test for (reverse) micelle formation can provide misleading conclusions and should be interpreted with care.

\section{Acknowledgements}

This work was financially supported by the Warsaw University of Technology. Experiments at the ISIS Pulsed Neutron and Muon Source were supported by a beamtime allocation from the Science and Technology Facilities Council. N. Fatin-Rouge is acknowledged for help in FCS measurements and A. Hoell for help in interpretation of SANS results.

\section{References}

1 K. Wojciechowski, M. Kucharek and J. Buffle, Mechanism of $\mathrm{Cu}(\mathrm{II})$ Transport through Permeation Liquid Membranes using Azacrown Ether and Fatty Acid as Carrierm, J. Membr. Sci., 2008, 314, 152-162.

2 Z. Zhang, J. Buffle, H. P. van Leeuwen and K. Wojciechowski, Roles of Metal Ion Complexation and Membrane Permeability in the Metal Flux through Lipophilic Membranes. Labile Complexes at Permeation Liquid Membranes, Anal. Chem., 2006, 78, 5693-5703.

3 J. Israelachvili, Intermolecular and Surface Force, Academic Press, 2011.

4 N. Parthasarathy and J. Buffle, Supported Liquid Membrane for Analytical Separation of Transition Metal Ions. Part I. Complexation Properties of 1,10-Didecyl-1,10-Diaza-18-Crown-6, Anal. Chim. Acta, 1991, 254, 1-7.

5 T. K. Hodgdon and E. W. Kaler, Hydrotropic Solutions, Curr. Opin. Colloid Interface Sci., 2007, 12, 121-128.

6 J. Eastoe, M. H. Hatzopoulos and P. J. Dowding, Action of Hydrotropes and Alkyl-Hydrotropes, Soft Matter, 2011, 7, 5917-5925.

7 P. Bauduin, F. Testard and T. Zemb, Solubilization in Alkanes by Alcohols as Reverse Hydrotropes Or "Lipotropes", J. Phys. Chem. B, 2008, 112, 12354-12360.

8 V. J. Gatto, K. A. Arnold, A. M. Viscariello, S. R. Miller, C. R. Morgan and G. W. Gokel, Syntheses and Binding Properties of Bibrachial Lariat Ethers (BiBLEs): survey of Synthetic Methods and Cation Selectivities, J. Org. Chem., 1986, 51, 5373-5384.

9 R. K. Heenan, J. Penfold and S. M. King, SANS at Pulsed Neutron Sources: present and Future Prospects, J. Appl. Crystallogr., 1997, 30, 1140-1147.

10 K. Wojciechowski and A. Brzozowska, From Gibbs- to Langmuir-Type Adsorbed Layers: alkylated Azacrown Ethers at Liquid-Liquid Interfaces, J. Phys. Chem. C, 2012, 116, 12584-12590.

11 V. Uskoković and M. Drofenik, Reverse Micelles: inert Nano-Reactors Or Physico-Chemically Active Guides of the Capped Reactions, Adv. Colloid Interface Sci., 2007, 133, 23-34.

12 M. P. Pileni, Reverse Micelles as Microreactors, J. Phys. Chem., 1993, 97, 6961-6973.

13 N. Basilio, L. García-Río and M. Martín-Pastor, NMR Evidence of Slow Monomer-Micelle Exchange in a CalixareneBased Surfactant, J. Phys. Chem. B, 2010, 114, 4816-4820.

14 P. S. Denkova, L. Van Lokeren and R. Willem, Mixed Micelles of Triton X-100, Sodium Dodecyl Dioxyethylene Sulfate, and Synperonic 161 Investigated by NOESY and Diffusion Ordered NMR Spectroscopy, J. Phys. Chem. B, 2009, 113, 6703-6709.

15 F. Asaro and N. Savko, Resolution of a Nonionic Surfactant Oligomeric Mixture by Means of DOSY with Inverse Micelle Assistance, Magn. Reson. Chem., 2011, 49, 195-198.

16 M. Holz, S. R. Heil and A. Sacco, Temperature-Dependent Self-Diffusion Coefficients of Water and Six Selected Molecular Liquids for Calibration in Accurate ${ }^{1} \mathrm{H}$ NMR PFG Measurements, Phys. Chem. Chem. Phys., 2000, 2, 4740-4742.

17 K. Wojciechowski and J. Buffle, The Interaction of Azacrown Ether with Fatty Acid in Nonpolar Solvents and at the Organic-Aqueous Interface, Biosens. Bioelectron., 2004, 20, 1051-1059.

18 S. Maccarrone, H. Frielinghaus, J. Allgaier, D. Richtery and P. Lindner, SANS Study of Polymer-Linked Droplets, Langmuir, 2007, 23, 9559-9562.

19 R. D. Neuman and T. H. Ibrahim, Novel Structural Model of Reversed Micelles: the Open Water-Channel Model, Langmuir, 1999, 15, 10-12.

20 R. Plehnert, J. A. Schroeter and C. Tschierske, Selective Cationic Binding at the Air-Water Interface by Thin Films of Rigid Amphiphiles Bearing Laterally Attached Crown Ether Moieties, Langmuir, 1998, 14, 5245-5249.

21 D. R. Rueda, A. Nogales, J. J. Hernández, M. GarcíaGutiérrez, T. A. Ezquerra, S. V. Roth, M. G. Zolotukhin and R. Serna, Stacking of Main Chain-Crown Ether Polymers in Thin Films, Langmuir, 2007, 23, 12677-12681.

22 K. M. Fromm, E. D. Gueneau, H. Goesmann and C. G. Bochet, "One-Dimensional Water" and "One-Dimensional Acid": synthesis and the Unique Crystal Structure of $\infty 1\left[\left(\mathrm{H}_{2} \mathrm{O}\right) \subset\right.$ $\left.(\mathrm{DB} 18 \mathrm{C} 6)\left(\mathrm{M} 2-\mathrm{H}_{2} \mathrm{O}\right)_{2 / 2}\right] \propto 1\left[\left(\mathrm{H}_{3} \mathrm{O}\right) \subset(\mathrm{DB} 18 \mathrm{C} 6)\left(\mathrm{M} 2-\mathrm{H}_{2} \mathrm{O}\right)_{2 / 2}\right] \mathrm{I}_{3}$ (DB18C6 = Dibenzo-18-Crown-6), Z. Anorg. Allg. Chem., 2003, 629, 597-600.

23 S. L. De Wall, L. J. Barbour and G. W. Gokel, Solid-State Bilayer Formation from a Dialkyl-Substituted Lariat Ether that Forms Stable Vesicles in Aqueous Suspension, J. Phys. Org. Chem., 2001, 14, 383-391.

24 R. M. Izatt, K. Pawlak, J. S. Bradshaw and R. L. Bruening, Thermodynamic and Kinetic Data for Macrocycle Interactions with Cations and Anions, Chem. Rev., 1991, 91, 1721-1785.

25 P. Kuzmič, Program DYNAFIT for the Analysis of Enzyme Kinetic Data: application to HIV Proteinase, Anal. Biochem., 1996, 237, 260-273. 